\title{
FIXED POINTS AND CONJUGACY CLASSES \\ OF REGULAR ELLIPTIC ELEMENTS IN $\operatorname{Sp}(3, \mathrm{Z})$
}

BY

\author{
MINKING EIE AND CHUNG - YUAN LIN
}

\begin{abstract}
In this paper, we obtain 13 isolated fixed points (up to a $\mathrm{Sp}(3, \mathbf{Z}$ )-equivalence) and 86 conjugacy classes of regular elliptic elements in $\operatorname{Sp}(3, \mathbf{Z})$. Hence the contributions from regular elliptic conjugacy classes in $\operatorname{Sp}(3, \mathbf{Z})$ to the dimension formula computed via the Selberg trace formula can be computed explictly by the main theorem of [4 or 5].
\end{abstract}

Introduction. In [6 and 7], E. Gottschling studied the fixed points and their isotropy groups of finite order elements in $\operatorname{Sp}(2, \mathbf{Z})$. He finally obtained six $\operatorname{Sp}(2, \mathbf{Z})$ inequivalent isolated fixed points as follows:

$$
\begin{aligned}
& Z_{1}=\operatorname{diag}[i, i], \\
& Z_{2}=\operatorname{diag}[\rho, \rho], \quad \rho=e^{\pi i / 3}, \\
& Z_{3}=\operatorname{diag}[i, \rho], \\
& Z_{4}=\frac{i}{\sqrt{3}}\left[\begin{array}{ll}
2 & 1 \\
1 & 2
\end{array}\right], \\
& Z_{5}=\left[\begin{array}{cc}
\eta & (\eta-1) / 2 \\
(\eta-1) / 2 & \eta
\end{array}\right], \quad \eta=\frac{1}{3}+\frac{2 \sqrt{2 i}}{3}, \\
& Z_{6}=\left[\begin{array}{cc}
\omega & \omega+\omega^{-2} \\
\omega+\omega^{-2} & -\omega^{-1}
\end{array}\right], \quad \omega=e^{2 \pi i / 5} .
\end{aligned}
$$

The isotropy subgroups at $Z_{i}(i=1,2,3,4,5,6)$ are groups of order 16, 36, 12, 12, 24 and 5 , respectively.

By the argument of [9], these fixed points can be obtained from symplectic embeddings of

$$
\begin{gathered}
Q(i) \oplus Q(i), \quad Q(\rho) \oplus Q(\rho), \quad Q(i) \oplus Q(\rho), \\
Q\left(e^{\pi i / 6}\right), \quad Q\left(e^{\pi i / 4}\right), \quad Q\left(e^{2 \pi i / 5}\right),
\end{gathered}
$$

into $M_{4}(Q)$. In this paper, we shall combine the reduction theory of symplectic matrices [2, 3] with the arguments of [8, 9] and obtain all $\operatorname{Sp}(3, \mathbf{Z})$-inequivalent isolated fixed point and conjugacy classes of regular elliptic elements in $\operatorname{Sp}(3, \mathbf{Z})$. A table for all representatives and their centralizer in $\operatorname{Sp}(3, \mathbf{Z}) /\{ \pm 1\}$ of regular elliptic conjugacy classes in $\operatorname{Sp}(3, \mathbf{Z})$ is given.

Received by the editors January 24, 1984.

1980 Mathematics Subject Classification. Primary 10D20; Secondary 10D05.

(1) 1985 American Mathematical Society $0002-9947 / 85 \$ 1.00+\$ .25$ per page 
1. Notations and basic results. Let $\mathbf{Z}, \mathbf{Q}, \mathbf{R}$ and $\mathbf{C}$ denote the ring of integers, the fields of rational, real and complex numbers, respectively. The real symplectic matrices of degree $n$,

$$
\operatorname{Sp}(n, \mathbf{R})=\left\{\left.M \in M_{2 n}(\mathbf{R})\right|^{t} M J M=J, J=\left[\begin{array}{cc}
0 & E_{n} \\
-E_{n} & 0
\end{array}\right]\right\},
$$

act on the generalized half space $H_{n}$ defined by

$$
H_{n}=\left\{Z \in M_{n}(\mathbf{C}) \mid Z={ }^{\prime} Z, \operatorname{Im} Z>0\right\} .
$$

Here $M_{2 n}(\mathbf{R})$ is the $2 n \times 2 n$ matrix ring over $\mathbf{R}, M_{n}(\mathbf{C})$ is the $n \times n$ matrix ring over C, $E_{n}$ is the identity of $M_{n}(\mathbf{C})$ and ${ }^{t} Z$ is the transpose of $Z$.

A point $Z_{0}$ in $H_{n}$ is called an isolated fixed point of $\operatorname{Sp}(3, \mathbf{Z})$ if there exists $M=\left[\begin{array}{ll}A & B \\ C & D\end{array}\right]$ in $\operatorname{Sp}(3, Z)$ such that $Z_{0}$ is the unique solution of the equation,

$$
A Z+B=Z(C Z+D), \quad Z \in H_{n} .
$$

An element $M$ of $\operatorname{Sp}(3, \mathbf{Z})$ is regular elliptic if $M$ has an isolated fixed point (see [4]). Now suppose $M$ is a regular elliptic element of $\operatorname{Sp}(3, \mathbf{Z})$; then by the discreteness of $\operatorname{Sp}(3, \mathbf{Z})$ and the property that $\operatorname{Sp}(3, \mathbf{Z})$ acts transitively on $H_{3}$, we conclude that

(1) $M$ is an element of finite order,

(2) $M$ is conjugate in $\operatorname{Sp}(3, \mathbf{R})$ to $\left[\begin{array}{cc}A & B \\ -B & A\end{array}\right]$ with $A+B i=\operatorname{diag}\left[\lambda_{1}, \lambda_{2}, \lambda_{3}\right], \lambda_{i}(i=$ $1,2,3)$ root of unity and $\lambda_{i} \lambda_{j} \neq 1$ for all $i, j$,

(3) the centralizer of $M$ in $\operatorname{Sp}(3, \mathbf{Z})$ is a group of finite order.

By property (1), we see that the minimal polynomial of $M$ is a product of different cyclotomic polynomials as follow: $X^{2}+1, X^{2}-X+1, X^{2}+X+1, X^{4}+1, X^{4}-$ $X^{2}+1, X^{4}+X^{3}+X^{2}+X+1, X^{4}-X^{3}+X^{2}-X+1, X^{6}-X^{3}+1, X^{6}+X^{3}$ $+1, X^{6}+X^{5}+X^{4}+X^{3}+X^{2}+X+1, X^{6}-X^{5}+X^{4}-X^{3}+X^{2}-X+1$.

For our convenience, we identify $\operatorname{Sp}\left(n_{1}, \mathbf{R}\right) \times \operatorname{Sp}\left(n_{2}, \mathbf{R}\right)$ as a subgroup of $\operatorname{Sp}\left(n_{1}+n_{2}, \mathbf{R}\right)$ via the embedding

$$
\left[\begin{array}{ll}
A & B \\
C & D
\end{array}\right] \times\left[\begin{array}{ll}
P & Q \\
R & S
\end{array}\right] \rightarrow\left[\begin{array}{cccc}
A & 0 & B & 0 \\
0 & P & 0 & Q \\
C & 0 & D & 0 \\
0 & R & 0 & S
\end{array}\right] .
$$

Also, we consider the unitary group $U(n)$ as a maximal compact subgroup of

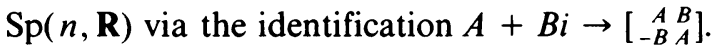

2. Reducible cases. For each regular elliptic element $M$ in $\operatorname{Sp}(3, \mathbf{Z})$, the $\operatorname{ring} Q(M)$ is isomorphic to a direct sum of cyclotomic fields which have degree at most 6 since $M$ is a semisimple element. The summand must be equal to one of the following:

$$
\begin{gathered}
Q\left[e^{\pi i / 2}\right], \quad Q\left[e^{2 \pi i / 3}\right], \quad Q\left[e^{\pi i / 4}\right], \quad Q\left[e^{2 \pi i / 5}\right], \\
Q\left[e^{\pi i / 6}\right], \quad Q\left[e^{2 \pi i / 7}\right], \quad Q\left[e^{2 \pi i / 9}\right] .
\end{gathered}
$$


Now suppose the characteristic polynomial $P(X)$ of $M$ is reducible over $\mathbf{Z}[X]$; then we obtain the following ten possible fixed points for $M$ simply from fixed points of regular elliptic elements of $\mathrm{SL}_{2}(\mathbf{Z})$ and $\mathrm{Sp}(2, \mathbf{Z})$.

1.

3.

$$
\begin{aligned}
& Z_{01}=\operatorname{diag}[i, i, i], \\
& Z_{04}=[i, \rho, \rho] \text {, } \\
& Z_{05}=\left[\begin{array}{ccc}
i & 0 & 0 \\
0 & \eta & (\eta-1) / 2 \\
0 & (\eta-1) / 2 & \eta
\end{array}\right], \quad \eta=\frac{1}{3}+\frac{2 \sqrt{2 i}}{3} \text {, }
\end{aligned}
$$

$Z_{03}=\operatorname{diag}[\rho, i, i]$,

5.

6. $\quad Z_{06}=\frac{i}{\sqrt{3}}\left[\begin{array}{ccc}\sqrt{3} & 0 & 0 \\ 0 & 2 & 1 \\ 0 & 1 & 2\end{array}\right]$,

7. $Z_{07}\left[\begin{array}{ccc}i & 0 & 0 \\ 0 & \omega & \omega+\omega^{-2} \\ 0 & \omega+\omega^{-2} & -\omega^{-1}\end{array}\right], \quad \omega=e^{2 \pi i / 5}$,

8. $Z_{08}=\left[\begin{array}{ccc}\rho & 0 & 0 \\ 0 & \eta & (\eta-1) / 2 \\ 0 & (\eta-1) / 2 & \eta\end{array}\right]$,

9. $\quad Z_{09}=\frac{i}{\sqrt{3}}=\left[\begin{array}{ccc}1+\bar{\rho} & 0 & 0 \\ 0 & 2 & 1 \\ 0 & 1 & 2\end{array}\right]$,

10.

$$
Z_{10}=\left[\begin{array}{ccc}
\rho & 0 & 0 \\
0 & \omega & \omega+\omega^{-2} \\
0 & \omega+\omega^{-2} & -\omega^{-1}
\end{array}\right]
$$

Let $G_{i}(i=01,02,03,04,05,06,07,08,09,10)$ be the isotropy group of $\operatorname{Sp}(3, Z) /\{ \pm 1\}$ at $Z_{i}(i=01,02,03,04,05,06,07,08,09,10)$, respectively. Then a direct calculation shows that the order of $G_{i}(i=01,02, \ldots, 10)$ are 192, 648, 96, $144,96,48,20,144,72,30$, respectively. By considering conjugacy classes in $G_{i}$ $(i=01,02, \ldots, 10)$, we get 72 conjugacy classes of regular elliptic elements of $\mathrm{Sp}(3, \mathrm{Z})$ as shown in the table.

Now we shall show that every regular elliptic element with reducible characteristic polynomial is conjugate in $\operatorname{Sp}(3, \mathbf{Z})$ to one of these 72 conjugacy classes. First we need

LEMma 1. Suppose $M \in \operatorname{Sp}(n, \mathbf{Z})$ with characteristic polynomial $P(X)$ satisfying

(1) $P(X)$ is a product of two relative prime polynomials $P_{1}(X)$ and $P_{2}(x)$ with integral coefficients of degrees $2 n_{1}$ and $2 n_{2}\left(n_{1}+n_{2}=n\right)$, respectively,

(2) $P_{i}(X)=X^{2 n_{i}} P_{i}(1 / X), i=1,2$.

Then there exists $R \in \mathrm{Sp}(n, Q)$ such that $R^{-1} M R=M_{1} \times M_{2} \in \operatorname{Sp}\left(n_{1}, Q\right) \times$ $\operatorname{Sp}\left(n_{2}, Q\right)$. Furthermore, the characteristic polynomial of $M_{1}\left(\right.$ resp. $\left.M_{2}\right)$ is $P_{1}(X)$ (resp. $P_{2}(X)$ ).

Proof. (See Lemmas 1 and 2 of [2].) 
Lemma 2. Let $M \in \mathrm{Sp}(n, \mathbf{Z})$. Suppose that there exists $R \in \operatorname{Sp}(n, Q)$ such that

$$
R^{-1} M R=\left[\begin{array}{cccc}
A & 0 & B & * \\
* & { }^{t} U & * & * \\
C & 0 & D & * \\
0 & 0 & 0 & U^{-1}
\end{array}\right]
$$

Then there exists $\tilde{R} \in \operatorname{Sp}(n, \mathbf{Z})$ such that $\tilde{R}^{-1} M \tilde{R}$ has the same form as $R^{-1} M R$.

Proof. (See Satz 2 of [3].)

TheOREM 1. Suppose $M$ is a regular elliptic element of $\operatorname{Sp}(3, \mathbf{Z})$ with a reducible characteristic polynomial $P(X)$. Then $M$ is conjugate in $\operatorname{Sp}(3, \mathbf{Z})$ to an element of $\bigcup_{i=01}^{10} G_{i}$.

Proof. Here we only prove three special cases, other cases follow with similar arguments.

(1) $P(X)=\left(X^{2}+1\right)^{3}$. A representative of $M$ in $U(3)$ is $\operatorname{diag}[i, i, i]$. Thus $M$ is conjugate in $\operatorname{Sp}(3, \mathbf{R})$ to $J=\left[\begin{array}{c}0 \\ -E\end{array}\right]$, i.e. there exists $L \in \operatorname{Sp}(3, \mathbf{R})$ such that $M=L^{-1} J L$. With the Iwasawa decomposition of $\operatorname{Sp}(3, \mathbf{R})$, we can write

$$
L=\left[\begin{array}{cc}
A & B \\
-B & A
\end{array}\right]\left[\begin{array}{cc}
U & S^{t} U^{-1} \\
0 & { }^{t} U^{-1}
\end{array}\right], \quad A+B i \in U(3) .
$$

Since $J$ commutes with $\left[\begin{array}{c}A \\ -B\end{array}\right]$, it follows

$$
M=\left[\begin{array}{cc}
U & S^{t} U^{-1} \\
0 & { }^{t} U^{-1}
\end{array}\right]^{-1} J=\left[\begin{array}{cc}
U & S^{t} U^{-1} \\
0 & { }^{t} U^{-1}
\end{array}\right] .
$$

This forces $U,{ }^{\prime} U^{-1}, S \in \mathrm{GL}(3, \mathbf{Z})$. Hence $M$ is conjugate in $\operatorname{Sp}(3, \mathbf{Z})$ to $J=\left[\begin{array}{cc}0 & E \\ -E & 0\end{array}\right]$.

(2) $P(X)=\left(X^{2}-X+1\right)^{3}$. A representative of $M$ in $U(3)$ is $\operatorname{diag}[\rho, \rho, \rho]$ or $\operatorname{diag}\left[\rho^{2}, \rho^{2}, \rho^{2}\right]$. On the other hand, $Q(M) \cong Q(\rho)$ as fields and the class number of $Q(\rho)$ is 1 by Theorem 11.1 in Chapter 11 of [10]. Hence the number of conjugacy classes of regular elliptic elements with $X^{2}-X+1$ as minimal polynomial is 2 by [8 or 9]. Thus $M$ is conjugate in $\operatorname{Sp}(3, \mathbf{Z})$ to $\left[\begin{array}{cc}E-E \\ E & 0\end{array}\right]$ or $\left.\left[\begin{array}{cc}E-E \\ E\end{array}\right]\right]^{2}$.

(3) $P(X)=\left(X^{2}+1\right)\left(X^{4}+X^{3}+X^{2}+X+1\right)$. Note that $M$ can be represented in $U(3)$ as

$$
e[1 / 2,2 / 5,4 / 5] \text { or } e[1 / 2,4 / 5,8 / 5] \text { or } e[1 / 2,6 / 5,2 / 5] \text { or } e[1 / 2,8 / 5,6 / 5] \text {. }
$$

$\left(e[a, b, c]\right.$ stands for $\left.\left[e^{\pi i a}, e^{\pi i b}, e^{\pi i c}\right]\right)$.

In particular, $M^{5}$ can be represented in $U(3)$ as $\operatorname{diag}[i, 1,1]$ or $[-i, 1,1]$ and has characteristic polynomial $\left(X^{2}+1\right)(X-1)^{4}$. By Lemmas 1 and 2 , there exists $R \in \operatorname{Sp}(3, \mathbf{Z})$ such that

$$
R^{-1} M^{5} R=\left[\begin{array}{cccc}
0 & 0 & 1 & * \\
* & E_{2} & * & * \\
-1 & 0 & 0 & * \\
0 & 0 & 0 & E_{2}
\end{array}\right] \text { or }\left[\begin{array}{cccc}
0 & 0 & -1 & * \\
* & E_{2} & * & * \\
1 & 0 & 0 & * \\
0 & 0 & 0 & E_{2}
\end{array}\right]
$$

which is conjugate in $\operatorname{Sp}(3, \mathbf{Z})$ to

$$
\left[\begin{array}{cc}
0 & 1 \\
-1 & 0
\end{array}\right] \times E_{4} \quad \text { or } \quad\left[\begin{array}{cc}
0 & -1 \\
1 & 0
\end{array}\right] \times E_{4} \text {. }
$$


Hence we may assume

$$
R^{-1} M^{5} R=\left[\begin{array}{cc}
0 & 1 \\
-1 & 0
\end{array}\right] \times E_{4} \quad \text { or } \quad\left[\begin{array}{cc}
0 & -1 \\
1 & 0
\end{array}\right] \times E_{4}
$$

Note that the isolated fixed point of $R^{-1} M R$ is contained in the set of fixed points of $R^{-1} M^{5} R$, i.e. the set

$$
Z=\left[\begin{array}{ccc}
i & 0 & 0 \\
0 & z_{2} & z_{23} \\
0 & z_{23} & z_{3}
\end{array}\right], \quad \operatorname{Im} Z>0
$$

Now it is easy to see that the isolated fixed point of $R^{-1} M R$ is $\left(\mathrm{SL}_{2}(\mathrm{Z}) \times \mathrm{Sp}(2, \mathrm{Z})\right)$ equivalent to $Z_{07}$ and $M$ is conjugate in $\operatorname{Sp}(3, \mathbf{Z})$ to an element of $G_{07}$. Q.E.D.

In the sections following, we shall determine conjugacy classes of regular elliptic elements of orders 9 and 7.

3. Symplectic embeddings of $Q\left(e^{2 \pi i / 9}\right)$ and $Q\left(e^{2 \pi i / 7}\right)$. For our convenience, we denote $e^{2 \pi i / 9}$ by $\zeta$. Note that $Q(\zeta)$ is the splitting field of the cyclotomic polynomial $X^{6}+X^{3}+1$ and contains the total real number field $Q\left(\zeta+\zeta^{-1}\right)$ which is the splitting field of $X^{3}-3 X+1$. By a symplectic embedding of $Q(\zeta)$ into $M_{6}(Q)$, we mean an injection from $Q(\zeta)$ into $M_{6}(Q)$ such that $\zeta$ is mapped into a symplectic matrix $M$ and $Q(\zeta) \cong Q(M)$ as fields [9].

LEMMA 3. Let $M$ be an element of $\operatorname{Sp}(3, \mathbf{Z})$ of order 9. Then $M$ is conjugate in $\operatorname{Sp}(3, \mathbf{R})$ to one of the following: $\left[\zeta, \zeta^{4}, \zeta^{7}\right],\left[\zeta, \zeta^{2}, \zeta^{4}\right],\left[\zeta, \zeta^{2}, \zeta^{5}\right],\left[\zeta, \zeta^{5}, \zeta^{7}\right],\left[\zeta^{2}, \zeta^{4}, \zeta^{8}\right]$, $\left[\zeta^{2}, \zeta^{5}, \zeta^{8}\right],\left[\zeta^{4}, \zeta^{7}, \zeta^{8}\right],\left[\zeta^{5}, \zeta^{7}, \zeta^{8}\right]$.

Proof. The minimal polynomial of $M$ is $X^{6}+X^{3}+1$ which can be factored into

$$
\begin{aligned}
& (X-\zeta)\left(X-\zeta^{2}\right)\left(X-\zeta^{4}\right)\left(X-\zeta^{5}\right)\left(X-\zeta^{7}\right)\left(X-\zeta^{8}\right) \\
& \quad=\left[X^{2}+\left(\zeta+\zeta^{-1}\right) X+1\right]\left[X^{2}-\left(\zeta^{2}+\zeta^{-2}\right) X+1\right]\left[X^{2}-\left(\zeta^{4}+\zeta^{-4}\right) X+1\right] .
\end{aligned}
$$

Hence $M$ is conjugate in $\operatorname{Sp}(3, \mathbf{R})$ to

$$
\left[\begin{array}{cc}
\cos \theta & \pm \sin \theta \\
\mp \sin \theta & \cos \theta
\end{array}\right] \times\left[\begin{array}{cc}
\cos 2 \theta & \pm \sin 2 \theta \\
\mp \sin 2 \theta & \cos 2 \theta
\end{array}\right] \times\left[\begin{array}{cc}
\cos 4 \theta & \pm \sin 4 \theta \\
\mp \sin 4 \theta & \cos 4 \theta
\end{array}\right], \quad \theta=\frac{2 \pi}{9} \text {. }
$$

Note that the above eight elements of $\operatorname{Sp}(3, \mathbf{R})$ are represented by the prescribed elements in $U(3)$ as in our lemma.

LEMMA 4. The number of conjugacy classes of regular elliptic elements of order 9 in $\mathrm{Sp}(3, \mathbf{Z})$ is 8 .

Proof. The ideal class number of $Q(\zeta)$ is 1 by Theorem 11.1 of [9], hence the number of conjugacy classes of regular elliptic elements of order 9 is given by [ $E_{0}: N(E)$ ], where

$E:$ the group of units in $Q(\zeta)$,

$E_{0}$ : the group of units in $Q\left(\zeta+\zeta^{-1}\right)$,

$N(E)=\{u \bar{u} \mid u \in E\}$,

according to the argument of [8 or 9 ].

The group of units for cyclotomic fields is determined in Chapter 8 of [10]. Applying this to our case, we get $\left[E_{0}: N(E)\right]=8$ when the cyclotomic field is $Q(\zeta)$. 
There are two conjugacy classes of elements of order 9 appearing in the isotropy group $G_{02}$ of $Z_{02}=\operatorname{diag}[\rho, \rho, \rho]$. Indeed, if we let $M=\left[\begin{array}{ll}A & B \\ C & D\end{array}\right]$ with

$$
\begin{aligned}
& A=\left[\begin{array}{lll}
0 & 0 & 0 \\
1 & 0 & 1 \\
0 & 1 & 0
\end{array}\right], \quad B=\left[\begin{array}{ccc}
0 & 0 & -1 \\
0 & 0 & 0 \\
0 & 0 & 0
\end{array}\right], \\
& C=\left[\begin{array}{lll}
0 & 0 & 1 \\
0 & 0 & 0 \\
0 & 0 & 0
\end{array}\right] \text { and } D=\left[\begin{array}{ccc}
0 & 0 & -1 \\
1 & 0 & 0 \\
0 & 0 & 0
\end{array}\right],
\end{aligned}
$$

then it is a direct verification to show that

(1) $M$ is an element of order 9.

(2) $M$ can be represented in $U(3)$ as $\left[\zeta, \zeta^{4}, \zeta^{7}\right]$ or $\left[\zeta^{2}, \zeta^{8}, \zeta^{5}\right]$,

(3) $M^{3}=\left[\begin{array}{l}0-E \\ E-E\end{array}\right]$ has an isolated fixed point at $Z_{02}$.

Now we begin to look for the other six conjugacy classes of regular elliptic elements of order 9 in $\operatorname{Sp}(3, \mathbf{Z})$.

THEOREM 2. Suppose $\alpha, \beta, \gamma$ are distinct roots of the equation $X^{3}-3 X+1=0$ (or more precisely, $\alpha=2 \cos 2 \pi / 9, \beta=2 \cos 4 \pi / 9, \gamma=2 \cos 8 \pi / 9)$,

$$
A=\left[\begin{array}{ccc}
0 & 1 & 0 \\
1 & -1 & 1 \\
0 & 1 & 1
\end{array}\right], \quad \Omega=\frac{1}{3}\left[\begin{array}{ccc}
-3+\alpha+\alpha^{2} & -3+\beta+\beta^{2} & -3+\gamma+\gamma^{2} \\
-1+\alpha^{2} & -1+\beta^{2} & -1+\gamma^{2} \\
1+\alpha & 1+\beta & 1+\gamma
\end{array}\right]
$$

and

$$
M=\left[\begin{array}{cc}
A & E \\
-E & 0
\end{array}\right]
$$

then

(1) $M$ is an element of order 9 in $\mathrm{Sp}(3, \mathbf{Z})$ and has an isolated fixed point at

$$
Z_{11}=-\frac{1}{2} A+i \Omega\left(E-\frac{1}{4}^{t} \Omega A^{2} \Omega\right)^{1 / 2}{ }^{t} \Omega
$$

(2) $M$ is conjugate in $\mathrm{Sp}(3, \mathbf{R})$ to $\left[\zeta, \zeta^{2}, \zeta^{4}\right]$ of $U(3)$,

(3) the centralizer of $M$ in $\mathrm{Sp}(3, \mathbf{Z}) /\{ \pm 1\}$ is a group of order 9 .

Proof. (1) Since the characteristic polynomial of $M$ is $X^{6}+X^{3}+1$, it follows that $M$ is an element of order 9 in $\operatorname{Sp}(3, \mathbf{Z})$. Note that $\frac{1}{3}^{t}\left[-3+\alpha+\alpha^{2},-1+\alpha^{2}\right.$, $1+\alpha]$ is the normalized eigenvector of $A$ corresponding to the eigenvalue $\alpha$. It follows that ${ }^{t} \Omega A \Omega=\operatorname{diag}[\alpha, \beta, \gamma]$ and

$$
\left(E-\frac{1}{4}^{t} \Omega A \Omega\right)^{1 / 2}=\operatorname{diag}\left[\left(1-\alpha^{2} / 4\right)^{1 / 2},\left(1-\beta^{2} / 4\right)^{1 / 2},\left(1-\gamma^{2} / 4\right)^{1 / 2}\right] .
$$

Now it is a direct verification to show that $A Z_{11}=Z_{11} A$ and $Z_{11}^{2}+A Z_{11}+E=0$. Thus $Z_{11}=-\frac{1}{2} A+i \Omega\left(E-\frac{1}{4}^{t} \Omega A^{2} \Omega\right)^{1 / 2} \Omega$ is a fixed point of $M$. But $M$ has exactly one fixed point by Lemma 3 , hence $Z_{11}$ is the unique isolated fixed point of $M$.

(2) Let $R=\left[\begin{array}{ll}\Omega & 0 \\ 0 & \Omega\end{array}\right]$. Then $R \in \operatorname{Sp}(3, \mathbf{R})$ and

$$
R^{-1} M R=\left[\begin{array}{cc}
\alpha & 1 \\
-1 & 0
\end{array}\right] \times\left[\begin{array}{cc}
\beta & 1 \\
-1 & 0
\end{array}\right] \times\left[\begin{array}{cc}
\gamma & 1 \\
-1 & 0
\end{array}\right]
$$

Note that $R^{-1} M R$ is conjugate in $\operatorname{Sp}(3, \mathbf{R})$ to

$$
\left[\begin{array}{cc}
\cos \theta & \sin \theta \\
-\sin \theta & \cos \theta
\end{array}\right] \times\left[\begin{array}{cc}
\cos 2 \theta & \sin 2 \theta \\
-\sin 2 \theta & \cos 2 \theta
\end{array}\right] \times\left[\begin{array}{cc}
\cos 3 \theta & \sin 3 \theta \\
-\sin 3 \theta & \cos 3 \theta
\end{array}\right], \quad \theta=\frac{2 \pi}{9},
$$


because $\left[\begin{array}{cc}2 \cos \mu & 1 \\ -1 & 0\end{array}\right]$ is conjugate in $\mathrm{SL}_{2}(\mathbf{R})$ to $\left[\begin{array}{cc}\cos \mu & \sin \mu \\ -\sin \mu \cos \mu & 0\end{array}\right.$. This proves our assertion in (2).

(3) Let $C(M, \mathbf{Z})$ be the centralizer of $M$ in $\operatorname{Sp}(3, \mathbf{Z}) /\{ \pm 1\}$. Suppose $\gamma$ is an element of $C(M, \mathbf{Z})$. Then

$$
M\left(\gamma\left(Z_{11}\right)\right)=\gamma\left(M\left(Z_{11}\right)\right)=\gamma\left(Z_{11}\right) .
$$

Since $Z_{11}$ is the only fixed point of $M$, this forces $\gamma\left(Z_{11}\right)=Z_{11}$.

Note that ${ }^{t} \Omega Z_{11} \Omega=R\left(Z_{11}\right)=\operatorname{diag}\left[-\bar{\zeta},-\bar{\zeta}^{2},-\bar{\zeta}^{4}\right]$. Here $R=\left[\begin{array}{l}\Omega \\ 0 \\ 0\end{array}\right]$ as in (2). From $\gamma\left(Z_{11}\right)=Z_{11}$, we get

$$
R \gamma R^{-1}\left({ }^{t} \Omega Z_{11} \Omega\right)={ }^{t} \Omega Z_{11} \Omega
$$

It follows that

$$
R \gamma R^{-1}=\left[\begin{array}{ll}
a & b \\
c & d
\end{array}\right] \times\left[\begin{array}{ll}
a^{\prime} & b^{\prime} \\
c^{\prime} & d^{\prime}
\end{array}\right] \times\left[\begin{array}{ll}
a^{\prime \prime} & b^{\prime \prime} \\
c^{\prime \prime} & d^{\prime \prime}
\end{array}\right]
$$

with

$$
\left\{\begin{array}{l}
-a \bar{\zeta}+b=c \bar{\zeta}^{2}-d \bar{\zeta}, \quad a d-b c=1, \\
-a^{\prime} \bar{\zeta}^{2}+b^{\prime}=c^{\prime} \bar{\zeta}^{4}-d^{\prime} \bar{\zeta}^{2}, \quad a^{\prime} d^{\prime}-b^{\prime} c^{\prime}=1, \\
-a^{\prime \prime} \bar{\zeta}^{4}+b^{\prime \prime}=c^{\prime \prime} \bar{\zeta}^{8}-d^{\prime \prime} \bar{\zeta}^{4}, \quad a^{\prime \prime} d^{\prime \prime}-b^{\prime \prime} c^{\prime \prime}=1 .
\end{array}\right.
$$

The general solution of $a, b, c, d$ is given by

$$
\left\{\begin{array}{l}
a=\cos \theta-\cot \frac{2 \pi}{9} \sin \theta, \quad b=-\sec \frac{2 \pi}{9} \sin \theta, \\
c=\sec \frac{2 \pi}{9} \sin \theta, \quad d=\cos \theta+\cot \frac{2 \pi}{9} \sin \theta,
\end{array} \quad \theta \in \mathbf{R} .\right.
$$

The characteristic polynomial of $\left[\begin{array}{ll}a & b \\ c & d\end{array}\right]$ is $X^{2}-2 \cos \theta X+1$, hence $2 \cos \theta$ is an algebraic integer of degree 1 or 3. On the other hand, the fact that $\gamma$ is an element of finite order implies $e^{i \theta}$ is a root of unity. Now we have the following cases:

Case I. If $2 \cos \theta$ is an algebraic integer of degree 3 , then the characteristic polynomial of $\gamma$ is an irreducible polynomial of degree 6. Since $M$ satisfies this case, the characteristic polynomial of $\gamma$ is $X^{6}+X^{3}+1$ or $X^{6}-X^{3}+1$. This leads to the fact that $\theta=2 \pi / 9$ or $4 \pi / 9$ or $8 \pi / 9$ and $\gamma$ is one of the following elements: $\pm M$, $\pm M^{2}, \pm M^{4}, \pm M^{5}, \pm M^{7}, \pm M^{8}$.

Case II. If $2 \cos \theta= \pm 1$, then $\gamma$ is an element of order 3. Then $\gamma= \pm M^{3}$ or $\pm M^{6}$ by a direct calculation.

Case III. If $2 \cos \theta= \pm 2$, then $\gamma= \pm E_{6}$.

Case IV. If $2 \cos \theta=0$, then

$$
\gamma=R^{-1}\left\{\left[\begin{array}{cc}
-\cot \eta & -\sec \eta \\
\sec \eta & \cot \eta
\end{array}\right] \times\left[\begin{array}{cc}
-\cot 2 \eta & -\sec 2 \eta \\
\sec 2 \eta & \cot 2 \eta
\end{array}\right] \times\left[\begin{array}{cc}
-\cot 4 \eta & -\sec 4 \eta \\
\sec 4 \eta & \cot 4 \eta
\end{array}\right]\right\} R
$$

with $\eta=2 \pi / 9$. Such a $\gamma$ is not an integral matrix. M.

By the above discussion, we conclude $C(M, \mathbf{Z})$ is a group of order 9 generated by 
TABLE. Regular elliptic conjugacy classes of $\operatorname{Sp}(3, \mathbf{Z})$

Here $e[a, b, c]$ stands for $\operatorname{diag}\left[e^{\pi i a}, e^{\pi i b}, e^{\pi i c}\right], P_{1}(X)=X^{4}+X^{3}+X^{2}+X+1$ and $P_{2}(X)=X^{6}+X^{5}+X^{4}+X^{3}+X^{2}+X+1$.

\begin{tabular}{|c|c|c|c|c|}
\hline No. & $\begin{array}{l}\text { Representative } \\
\quad \text { in } U(3)\end{array}$ & $\begin{array}{c}\text { Minimal } \\
\text { polynomial }\end{array}$ & $\begin{array}{l}\text { Order of } \\
\text { centralizer }\end{array}$ & $\begin{array}{l}\text { No. of conjugates } \\
\text { in isotropy group }\end{array}$ \\
\hline 1 & $e[1 / 2,1 / 2,1 / 2]$ & $X^{2}+1$ & 192 & 1 \\
\hline 2 & $e[1 / 2,1 / 4,5 / 4]$ & $\left(X^{2}+1\right)\left(X^{4}+1\right)$ & 16 & 12 \\
\hline 3 & $e[1 / 2,3 / 4,7 / 4]$ & $\left(X^{2}+1\right)\left(X^{4}+1\right)$ & 16 & 12 \\
\hline 4 & $e[1 / 6,5 / 6,9 / 6]$ & $X^{6}+1$ & 6 & 32 \\
\hline 5 & $e[1 / 3,1 / 3,1 / 3]$ & $X^{2}-X+1$ & 648 & 1 \\
\hline 6 & $e[2 / 3,1 / 3,1 / 3]$ & $X^{4}+X^{2}+1$ & 216 & 3 \\
\hline 7 & $e[4 / 3,1 / 3,1 / 3]$ & $X^{4}+X^{2}+1$ & 216 & 3 \\
\hline 8 & $e[2 / 3,2 / 3,1 / 3]$ & $X^{4}+X^{2}+1$ & 216 & 3 \\
\hline 9 & $e[2 / 3,2 / 3,2 / 3]$ & $X^{2}+X+1$ & 648 & 1 \\
\hline 10 & $e[5 / 3,2 / 3,2 / 3]$ & $X^{4}+X^{2}+1$ & 216 & 3 \\
\hline 11 & $e[1 / 3,1 / 3,4 / 3]$ & $X^{4}+X^{2}+1$ & 36 & 18 \\
\hline 12 & $e[2 / 3,2 / 3,5 / 3]$ & $X^{4}+X^{2}+1$ & 36 & 18 \\
\hline 13 & $e[1 / 3,1 / 6,7 / 6]$ & $X^{4}+X^{2}+1$ & 36 & 18 \\
\hline 14 & $e[2 / 3,1 / 6,7 / 6]$ & $X^{4}+X^{2}+1$ & 36 & 18 \\
\hline 15 & $e[1 / 3,5 / 6,11 / 6]$ & $X^{4}+X^{2}+1$ & 36 & 18 \\
\hline 16 & $e[2 / 3,5 / 6,11 / 6]$ & $X^{4}+X^{2}+1$ & 36 & 18 \\
\hline 17 & $e[2 / 9,8 / 9,14 / 9]$ & $X^{6}+X^{3}+1$ & 9 & 72 \\
\hline 18 & $e[4 / 9,10 / 9,16 / 9]$ & $X^{6}+X^{3}+1$ & 9 & 72 \\
\hline 19 & $e[1 / 3,1 / 2,1 / 2]$ & $\left(X^{2}-X+1\right)\left(X^{2}+1\right)$ & 96 & 1 \\
\hline 20 & $e[2 / 3,1 / 2,1 / 2]$ & $\left(X^{2}+X+1\right)\left(X^{2}+1\right)$ & 96 & 1 \\
\hline 21 & $e[4 / 3,1 / 2,1 / 2]$ & $\left(X^{2}+X+1\right)\left(X^{2}+1\right)$ & 96 & 1 \\
\hline 22 & $e[5 / 3,1 / 2,1 / 2]$ & $\left(X^{2}-X+1\right)\left(X^{2}+1\right)$ & 96 & 1 \\
\hline 23 & $e[1 / 3,1 / 4,5 / 4]$ & $\left(X^{2}-X+1\right)\left(X^{4}+1\right)$ & 24 & 4 \\
\hline 24 & $e[2 / 3,1 / 4,5 / 4]$ & $\left(X^{2}+X+1\right)\left(X^{4}+1\right)$ & 24 & 4 \\
\hline 25 & $e[1 / 3,3 / 4,7 / 4]$ & $\left(X^{2}-X+1\right)\left(X^{4}+1\right)$ & 24 & 4 \\
\hline 26 & $e[2 / 3,3 / 4,7 / 4]$ & $\left(X^{2}+X+1\right)\left(X^{4}+1\right)$ & 24 & 4 \\
\hline 27 & $e[1 / 2,1 / 3,1 / 3]$ & $\left(X^{2}+1\right)\left(X^{2}-X+1\right)$ & 144 & 1 \\
\hline 28 & $e[3 / 2,1 / 3,1 / 3]$ & $\left(X^{2}+1\right)\left(X^{2}-X+1\right)$ & 144 & 1 \\
\hline 29 & $e[1 / 2,2 / 3,2 / 3]$ & $\left(X^{2}+1\right)\left(X^{2}-X+1\right)$ & 144 & 1 \\
\hline 30 & $e[3 / 2,2 / 3,2 / 3]$ & $\left(X^{2}+1\right)\left(X^{2}+X+1\right)$ & 144 & 1 \\
\hline 31 & $e[1 / 2,2 / 3,1 / 3]$ & $\left(X^{2}+1\right)\left(X^{4}+X^{2}+1\right)$ & 72 & 2 \\
\hline 32 & $e[3 / 2,2 / 3,1 / 3]$ & $\left(X^{2}+1\right)\left(X^{4}+X^{2}+1\right)$ & 72 & 2 \\
\hline 33 & $e[1 / 2,4 / 3,1 / 3]$ & $\left(X^{2}+1\right)\left(X^{4}+X^{2}+1\right)$ & 72 & 2 \\
\hline 34 & $e[1 / 2,5 / 3,2 / 3]$ & $\left(X^{2}+1\right)\left(X^{4}+X^{2}+1\right)$ & 72 & 2 \\
\hline 35 & $e[1 / 2,1 / 6,7 / 6]$ & $\left(X^{2}+1\right)\left(X^{4}-X^{2}+1\right)$ & 24 & 6 \\
\hline 36 & $e[1 / 2,1 / 3,4 / 3]$ & $\left(X^{2}+1\right)\left(X^{4}+X^{2}+1\right)$ & 24 & 6 \\
\hline 37 & $e[1 / 2,5 / 6,11 / 6]$ & $\left(X^{2}+1\right)\left(X^{4}-X^{2}+1\right)$ & 24 & 6 \\
\hline 38 & $e[1 / 2,2 / 3,5 / 3]$ & $\left(X^{2}+1\right)\left(X^{4}+X^{2}+1\right)$ & 24 & 6 \\
\hline 39 & $e[1 / 2,1 / 4,3 / 4]$ & $\left(X^{2}+1\right)\left(X^{4}+1\right)$ & 16 & 6 \\
\hline 40 & $e[1 / 2,5 / 4,7 / 4]$ & $\left(X^{2}+1\right)\left(X^{4}+1\right)$ & 16 & 6 \\
\hline 41 & $e[1 / 2,1 / 3,2 / 3]$ & $\left(X^{2}+1\right)\left(X^{4}+X^{2}+1\right)$ & 24 & 2 \\
\hline 42 & $e[3 / 2,1 / 3,2 / 3]$ & $\left(X^{2}+1\right)\left(X^{2}-X+1\right)$ & 24 & 2 \\
\hline
\end{tabular}


TABLE (continued)

\begin{tabular}{|c|c|c|c|c|}
\hline No. & $\begin{array}{l}\text { Representative } \\
\quad \text { in } U(3)\end{array}$ & $\begin{array}{c}\text { Minimal } \\
\text { polynomial }\end{array}$ & $\begin{array}{l}\text { Order of } \\
\text { centralizer }\end{array}$ & $\begin{array}{l}\text { No. of conjugates } \\
\text { in isotropy group }\end{array}$ \\
\hline 43 & $e[1 / 2,2 / 5,4 / 5]$ & $\left(X^{2}+1\right) P_{1}(X)$ & 20 & 1 \\
\hline 44 & $e[1 / 2,4 / 5,8 / 5]$ & $\left(X^{2}+1\right) P_{1}(X)$ & 20 & 1 \\
\hline 45 & $e[1 / 2,6 / 5,2 / 5]$ & $\left(X^{2}+1\right) P_{1}(X)$ & 20 & 1 \\
\hline 46 & $e[1 / 2,8 / 5,6 / 5]$ & $\left(X^{2}+1\right) P_{1}(X)$ & 20 & 1 \\
\hline 47 & $e[3 / 2,2 / 5,4 / 5]$ & $\left(X^{2}+1\right) P_{1}(-X)$ & 20 & 1 \\
\hline 48 & $e[3 / 2,4 / 5,8 / 5]$ & $\left(X^{2}+1\right) P_{1}(-X)$ & 20 & 1 \\
\hline 49 & $e[3 / 2,6 / 5,2 / 5]$ & $\left(X^{2}+1\right) P_{1}(-X)$ & 20 & 1 \\
\hline 50 & $e[3 / 2,8 / 5,6 / 5]$ & $\left(X^{2}+1\right) P_{1}(-X)$ & 20 & 1 \\
\hline 51 & $e[1 / 3,1 / 4,3 / 4]$ & $\left(X^{2}-X+1\right)\left(X^{4}+1\right)$ & 24 & 6 \\
\hline 52 & $e[2 / 3,1 / 4,3 / 4]$ & $\left(X^{2}-X+1\right)\left(X^{4}+1\right)$ & 24 & 6 \\
\hline 53 & $e[4 / 3,1 / 4,3 / 4]$ & $\left(X^{2}+X+1\right)\left(X^{4}+1\right)$ & 24 & 6 \\
\hline 54 & $e[5 / 3,1 / 4,3 / 4]$ & $\left(X^{2}+X+1\right)\left(X^{4}+1\right)$ & 24 & 6 \\
\hline 55 & $e[1 / 3,1 / 3,2 / 3]$ & $X^{4}+X^{2}+1$ & 36 & 4 \\
\hline 56 & $e[2 / 3,1 / 3,2 / 3]$ & $X^{4}+X^{2}+1$ & 36 & 4 \\
\hline 57 & $e[1 / 3,2 / 5,4 / 5]$ & $\left(X^{2}-X+1\right) P_{1}(X)$ & 30 & 1 \\
\hline 58 & $e[2 / 3,2 / 5,4 / 5]$ & $\left(X^{2}+X+1\right) P_{1}(X)$ & 30 & 1 \\
\hline 59 & $e[4 / 3,2 / 5,4 / 5]$ & $\left(X^{2}+X+1\right) P_{1}(X)$ & 30 & 1 \\
\hline 60 & $e[5 / 3,2 / 5,4 / 5]$ & $\left(X^{2}-X+1\right) P_{1}(X)$ & 30 & 1 \\
\hline 61 & $e[1 / 3,4 / 5,8 / 5]$ & $\left(X^{2}-X+1\right) P_{1}(X)$ & 30 & 1 \\
\hline 62 & $e[2 / 3,4 / 5,8 / 5]$ & $\left(X^{2}+X+1\right) P_{1}(X)$ & 30 & 1 \\
\hline 63 & $e[4 / 3,4 / 5,8 / 5]$ & $\left(X^{2}+X+1\right) P_{1}(X)$ & 30 & 1 \\
\hline 64 & $e[5 / 3,4 / 5,8 / 5]$ & $\left(X^{2}-X+1\right) P_{1}(X)$ & 30 & 1 \\
\hline 65 & $e[1 / 3,6 / 5,2 / 5]$ & $\left(X^{2}-X+1\right) P_{1}(X)$ & 30 & 1 \\
\hline 66 & $e[2 / 3,6 / 5,2 / 5]$ & $\left(X^{2}+X+1\right) P_{1}(X)$ & 30 & 1 \\
\hline 67 & $e[4 / 3,6 / 5,2 / 5]$ & $\left(X^{2}+X+1\right) P_{1}(X)$ & 30 & 1 \\
\hline 68 & $e[5 / 3,6 / 5,2 / 5]$ & $\left(X^{2}-X+1\right) P_{1}(X)$ & 30 & 1 \\
\hline 69 & $e[1 / 3,8 / 5,6 / 5]$ & $\left(X^{2}-X+1\right) P_{1}(X)$ & 30 & 1 \\
\hline 70 & $e[2 / 3,8 / 5,6 / 5]$ & $\left(X^{2}+X+1\right) P_{1}(X)$ & 30 & 1 \\
\hline 71 & $e[4 / 3,8 / 5,6 / 5]$ & $\left(X^{2}+X+1\right) P_{1}(X)$ & 30 & 1 \\
\hline 72 & $e[5 / 3,8 / 5,6 / 5]$ & $\left(X^{2}-X+1\right) P_{1}(X)$ & 30 & 1 \\
\hline 73 & $e[2 / 9,4 / 9,8 / 9]$ & $X^{6}+X^{3}+1$ & 9 & 1 \\
\hline 74 & $e[4 / 9,8 / 9,16 / 9]$ & $X^{6}+X^{3}+1$ & 9 & 1 \\
\hline 75 & $e[8 / 9,16 / 9,14 / 9]$ & $X^{6}+X^{3}+1$ & 9 & 1 \\
\hline 76 & $e[10 / 9,2 / 9,4 / 9]$ & $X^{6}+X^{3}+1$ & 9 & 1 \\
\hline 77 & $e[14 / 9,10 / 9,2 / 9]$ & $X^{6}+X^{3}+1$ & 9 & 1 \\
\hline 78 & $e[16 / 9,14 / 9,10 / 9]$ & $X^{6}+X^{3}+1$ & 9 & 1 \\
\hline 79 & $e[2 / 7,4 / 7,6 / 7]$ & $P_{2}(X)$ & 7 & 1 \\
\hline 80 & $e[4 / 7,8 / 7,12 / 7]$ & $P_{2}(X)$ & 7 & 1 \\
\hline 81 & $e[6 / 7,12 / 7,4 / 7]$ & $P_{2}(X)$ & 7 & 1 \\
\hline 82 & $e[8 / 7,2 / 7,10 / 7]$ & $P_{2}(X)$ & 7 & 1 \\
\hline 83 & $e[10 / 7,6 / 7,2 / 7]$ & $P_{2}(X)$ & 7 & 1 \\
\hline 84 & $e[12 / 7,10 / 7,8 / 7]$ & $P_{2}(X)$ & 7 & 1 \\
\hline 85 & $e[2 / 7,4 / 7,8 / 7]$ & $P_{2}(X)$ & 7 & 3 \\
\hline 86 & $e[6 / 7,12 / 7,10 / 7]$ & $P_{2}(X)$ & 7 & 3 \\
\hline
\end{tabular}


With the same argument, we get the following result by simply replacing the role of $e^{2 \pi i / 9}$ by $e^{2 \pi i / 7}$.

Lemma 5. Let $M$ be an element of $\mathrm{Sp}(3, \mathbf{Z})$ of order 7 . Then $M$ is conjugate in $\operatorname{Sp}(3, \mathbf{R})$ to one of the following $\left(v=e^{2 \pi i / 7}\right)$ :

$$
\begin{aligned}
& {\left[v, v^{2}, v^{3}\right], \quad\left[v, v^{2}, v^{4}\right], \quad\left[v, v^{4}, v^{5}\right], \quad\left[v, v^{3}, v^{5}\right],} \\
& {\left[v^{2}, v^{3}, v^{6}\right], \quad\left[v^{2}, v^{4}, v^{6}\right], \quad\left[v^{4}, v^{5}, v^{6}\right], \quad\left[v^{3}, v^{5}, v^{6}\right] .}
\end{aligned}
$$

LEMMA 6. The number of conjugacy classes of regular elliptic elements of order 7 in $\operatorname{Sp}(3, \mathbf{Z})$ is 8 .

THEOREM 3. Suppose $\alpha, \beta, \gamma$ are distinct roots of the equation $X^{3}+X^{2}-2 X+1$ (or more precisely, $\alpha=2 \cos 2 \pi / 7, \beta=2 \cos 4 \pi / 7, \gamma=2 \cos 6 \pi / 7$ ),

$$
B=\left[\begin{array}{ccc}
0 & 1 & 0 \\
1 & 0 & 1 \\
0 & 1 & -1
\end{array}\right], \quad \Omega^{\prime}=\left[\begin{array}{ccc}
\frac{\alpha+\alpha^{2}}{1+3 \alpha} & \frac{\beta+\beta^{2}}{1+3 \beta} & \frac{\gamma+\gamma^{2}}{1+3 \gamma} \\
\frac{1+2 \alpha}{1+3 \alpha} & \frac{1+2 \beta}{1+3 \beta} & \frac{1+2 \gamma}{1+3 \gamma} \\
\frac{\alpha^{2}}{1+3 \alpha} & \frac{\beta^{2}}{1+3 \beta} & \frac{\gamma^{2}}{1+3 \gamma}
\end{array}\right]
$$

and

$$
M=\left[\begin{array}{cc}
B & E \\
-E & 0
\end{array}\right]
$$

Then

(1) $M$ is an element of order 7 in $\mathrm{Sp}(3, \mathbf{Z})$ and has an isolated fixed point at

$$
Z_{12}=-\frac{1}{2} B+i \Omega^{\prime}\left(E-\frac{1}{4}^{t} \Omega^{\prime} B^{2} \Omega^{\prime}\right)^{1 / 2} \Omega^{\prime}
$$

(2) $M$ is conjugate in $\mathrm{Sp}(3, \mathbf{R})$ to $\left[v, v^{2}, v^{3}\right]$,

(3) the centralizer of $M$ in $\operatorname{Sp}(3, \mathbf{Z}) /\{ \pm 1\}$ is a group of order 7 generated by $M$.

Note that $\left[v, v^{2}, v^{4}\right]$ and $\left[v^{3}, v^{6}, v^{5}\right]$ are exclusive in the set of all powers of $\left[v, v^{2}, v^{3}\right]$. To find all representatives for elliptic conjugacy classes of order 7 , it suffices to get a representative which is conjugate in $\operatorname{Sp}(3, \mathbf{R})$ to $\left[v, v^{2}, v^{4}\right]$.

THEOREM 4. Let $B, \Omega^{\prime}$ be matrices as in Theorem 3,

$$
U=\operatorname{diag}[1,1,-1] \text { and } M=\left[\begin{array}{cc}
B & E+B \\
-(E+B)^{-1} & 0
\end{array}\right]
$$

Then

(1) $M$ is an element of order 7 in $\operatorname{Sp}(3, \mathbf{Z})$ with isolated fixed point at

$$
Z_{13}=-\frac{1}{2} B(B+E)+i \Omega^{\prime}\left[\left(E-\frac{1}{4}^{t} \Omega^{\prime} B^{2} \Omega^{\prime}\right)^{1 / 2} \Omega^{\prime}(B+E) \Omega U\right]^{t} \Omega^{\prime},
$$

(2) $M$ is conjugate in $\mathrm{Sp}(3, \mathbf{R})$ to $\left[v, v^{2}, v^{4}\right]$,

(3) the centralizer of $M$ in $\operatorname{Sp}(3, \mathbf{Z}) /\{ \pm 1\}$ is a finite group of order 7 generated by $M$. 
Proof. Since $\operatorname{det}(E+B)=1$ and

$$
{ }^{t} \Omega^{\prime}(E+B) \Omega^{\prime}=\operatorname{diag}\left[1+2 \cos \frac{2 \pi}{7}, 1+2 \cos \frac{4 \pi}{7}, 1+2 \cos \frac{6 \pi}{7}\right]
$$

has signature,,++- , it follows that $M \in \operatorname{Sp}(3, \mathbf{Z})$ and $M$ is conjugate in $\operatorname{Sp}(3, \mathbf{R})$ to

$$
M^{\prime}=\left[\begin{array}{cc}
2 \cos \theta & 1 \\
-1 & 0
\end{array}\right] \times\left[\begin{array}{cc}
2 \cos 2 \theta & 1 \\
-1 & 0
\end{array}\right] \times\left[\begin{array}{cc}
2 \cos 3 \theta & -1 \\
1 & 0
\end{array}\right], \quad \theta=\frac{2 \pi}{7} .
$$

Indeed, if we let $R^{\prime}=\Omega^{\prime} \Lambda$ with

$$
\Lambda=\operatorname{diag}\left[\left(1+2 \cos \frac{2 \pi}{7}\right)^{-1 / 2},\left(1+2 \cos \frac{2 \pi}{7}\right)^{-1 / 2},\left(-1-2 \cos \frac{2 \pi}{7}\right)^{-1 / 2}\right]
$$

then $\left(R^{\prime}\right)^{-1} M R^{\prime}=M^{\prime}$. Hence (1) and (2) follow as a direct calculation. By a similar argument as in (3) of Theorem 2, we get (2).

By Theorems 1, 2, 3 and 4, we obtain the following table for conjugacy classes of regular elliptic elements in $\operatorname{Sp}(3, \mathbf{Z})$.

4. Application. Contributions from conjugacy classes of regular elliptic elements in $\operatorname{Sp}(n, \mathbf{Z})$ to the dimension formula for Siegel cusp forms of degree $n$ and weight $k$ [4] are given by

$$
\sum|C(M, \mathbf{Z})|^{-1} \prod_{i=1}^{n} \bar{\lambda}_{i}^{k} \prod_{i \leq j}\left(1-\bar{\lambda}_{i} \bar{\lambda}_{j}\right)^{-1}
$$

Here the summation in $M$ ranges over all conjugacy classes of regular elliptic elements in $\operatorname{Sp}(n, \mathbf{Z}) . M$ is conjugate in $\operatorname{Sp}(n, \mathbf{R})$ to $\left[{ }_{-B A}^{A}{ }_{A}^{B}\right]$ with $A+B i=$ $\operatorname{diag}\left[\lambda_{1}, \lambda_{2}, \ldots, \lambda_{n}\right], \lambda_{i} \lambda_{j} \neq 1$ for all $i, j$ and $C(M, \mathbf{Z})$ is the centralizer of $M$ in $\operatorname{Sp}(3, \mathbf{Z})$. Applying this formula to the case $n=3$, we get all contributions from 86 regular elliptic conjugacy classes in $\operatorname{Sp}(3, \mathbf{Z})$.

For the case $n=1$ and $n=2$, the contribution from a particular regular elliptic conjugacy class appears to be a residue of a generating function at a simple pole. For example, the contribution from the conjugacy class of regular elliptic elements of order 5 in $\operatorname{Sp}(2, \mathbf{Z})$ is given by

$$
\begin{aligned}
& K=\frac{1}{25}\left[\omega^{-6 k}\left(1-\omega^{-2}\right)+\omega^{-2 k}\left(1-\omega^{-4}\right)+\omega^{-8 k}\left(1-\omega^{-6}\right)+\omega^{-4 k}\left(1-\omega^{-8}\right)\right], \\
& \text { which is precisely the negative of the sum of residues of the function }
\end{aligned}
$$

$$
\frac{1}{\left(1-T^{4}\right)\left(1-T^{6}\right)\left(1-T^{10}\right)\left(1-T^{12}\right) T^{k+1}}
$$

at $T=e^{i \theta}$ with $\theta= \pm \pi / 5, \pm 2 \pi / 5, \pm 3 \pi / 5, \pm 4 \pi / 5$ when $k$ is even.

It is easy to see that the total contribution from conjugacy classes of elements of order 2 or 3 in $\mathrm{SL}_{2}(\mathrm{Z})$ is the negative of the sum of residues of the function

$$
\frac{1}{\left(1-T^{4}\right)\left(1-T^{6}\right) T^{k+1}}
$$

at $T=e^{i \theta}$ with $\theta= \pm \pi / 2, \pm \pi / 3, \pm 2 \pi / 3$ when $k$ is even. 
Note that

$$
\frac{1}{\left(1-T^{4}\right)\left(1-T^{6}\right)} \text { and } \frac{1}{\left(1-T^{4}\right)\left(1-T^{6}\right)\left(1-T^{10}\right)\left(1-T^{12}\right)}
$$

are well known to be generating functions of dimension formulas for modular forms of degree 1 and degree 2, respectively. It is hopeful to find a generating function of a dimension formula for modular forms of degree 3 by computing contributions from conjugacy classes of regular elliptic elements in $\operatorname{Sp}(3, \mathbf{Z})$. However, we can write down explictly the conjugacy classes of $\operatorname{Sp}(3, \mathbf{Z})$ simply by using our results in this paper and reduction theory in $[2,3]$. Thus a dimension formula for Siegel cusps forms of degree 3 can be obtained by the Selberg trace formula and results of [5].

\section{REFERENCES}

1. Z. I. Borevich and I. R. Shafarevich, Number theory, Academic Press, New York, 1966.

2. U. Christian, A reduction theory for symplectic matrices, Math. Z. 101 (1967), 213-244.

3. __ Zur Theorie der symplektischen Gruppen, Acta Arith. 24 (1973), 61-85.

4. Minking Eie, Contributions from conjugacy classes of regular elliptic elements in $\operatorname{Sp}(n, \mathbf{Z})$ to the dimension formula, Trans. Amer. Math. Soc. 285 (1984), 403-410.

5. Siegel cusp forms of degree two and three, Mem. Amer. Math. Soc. (to appear).

6. E. Gottschling, Über die Fixpunkte der Siegelschen Modulgruppe, Math. Ann. 143 (1961), 111-149.

7. . Über die Fixpunktuntergruppen der Siegelschen Modulgruppe, Math. Ann. 143 (1961), $399-430$.

8. H. Midorikawa, On the number of regular elliptic conjugacy classes in the Siegel modular group of degree $2 n$. Tokyo J. Math. 6 (1983), 25-28.

9. B. Steinle, Fixpunktmannigfaltigkeiten symplektischen Matrizen, Acta Arith. 20 (1972), 63-106.

10. Lawrence C. Washington, Introduction to cyclotomic fields, Springer-Verlag, New York, 1982.

Institute of Mathematics, Academia Sinica, Nankang, Taipei, Taiwan, Republic of China

Department of Mathematics, National Tsing - Hua University, Hsinchu, Taiwan, Republic OF China (Current address of Chuang-Yuan Lin)

Current address (Minking Eie): Sonderforschungsbereich 170, Mathematisches Institut der GeorgAugust-Universität, Göttingen, Bunsenstrasse 3-5, D-3400 Göttingen, Federal Republic of Germany 